\title{
RIGIDITY OF A TRACE ESTIMATE FOR STEKLOV EIGENVALUES
}

\author{
YONGJIE SHI ${ }^{1}$ AND CHENGJIE YU ${ }^{2}$
}

\begin{abstract}
In this short note, we show the rigidity of a trace estimate for Steklov eigenvalues with respect to functions in our previous work (Trace and inverse trace of Steklov eigenvalues. J. Differential Equations 261 (2016), no. 3, 2026-2040.). Namely, we show that equality of the estimate holds if and only if the manifold is a direct product of a round ball and a closed manifold. The key ingredient in the proof is a decomposition theorem for flat and totally geodesic Riemannian submersions which may be of independent interests.
\end{abstract}

\section{INTRODUCTION}

Let $\left(M^{n}, g\right)$ be a compact Riemannian manifold with nonempty boundary. If the following boundary value problem:

$$
\left\{\begin{array}{l}
\Delta u=0 \\
\frac{\partial u}{\partial \nu}=\sigma u
\end{array}\right.
$$

has a nontrivial solution, then we call the constant $\sigma$ a Steklov eigenvalue of $(M, g)$. Here $\nu$ is the unit outward normal vector field on $\partial M$. The Rayleigh quotient corresponding to Steklov eigenvalues is

$$
Q(u)=\frac{\int_{M}\|\nabla u\|^{2} d V_{M}}{\int_{\partial M} u^{2} d V_{\partial M}} .
$$

Steklov [15, 9] considered this kind of eigenvalue problems because it is closely related to the frequency of liquid sloshing in a container. It is not hard to see that Steklov eigenvalues are just eigenvalues of the Dirichlet-to-Neumann map that sends Dirichlet boundary data of a harmonic function on $M$ to its Neumann boundary data. Steklov eigenvalues were extensively study in the past decades, because it is deeply related to free boundary minimal submanifolds and conformal

2010 Mathematics Subject Classification. Primary 35P15; Secondary 58J32.

Key words and phrases. Steklov eigenvalue, development, submersion.

${ }^{1}$ Research partially supported by NSF of China with contract no. 11701355 .

${ }^{2}$ Research partially supported by NSF of China with contract no. 11571215 . 
geometry in differential geometry $([4,5])$, liquid sloshing in physics and Calderón inverse problem ([3, 16]) in applied mathematics.

Higher order Dirichlet-to-Neumann maps were also considered in literature [1, 7], because they are closely related to inverse problems for the Maxwell equation in electromagnetics. However, the Dirichlet-toNeumann maps considered in [1, 7] was not suitable for spectral analysis. In 2012, Raulot and Savo [12] introduced a new notion of higher order Dirichlet-to-Neumann maps which is suitable for spectral analysis.

We would also like to mention that discrete versions of the classical Dirichlet-to-Neumann maps and higher order Dirichlet-to-Neuman maps were introduced in [6] and [14] respectively.

The Steklov eigenvalues of a Riemannian manifold $(M, g)$ can be listed in ascending order counting multiplicity as follows:

$$
0=\sigma_{0}<\sigma_{1} \leq \sigma_{2} \leq \cdots \leq \sigma_{k} \leq \cdots .
$$

In [13, by further extending the idea of Raulot-Savo in [11, 12, among the others, we obtained the following trace estimate for Steklov eigenvalues:

Theorem 1.1. Let $\left(M^{n}, g\right)$ be a compact Riemannian manifold with nonempty boundary and $V$ be the space of parallel exact 1-forms on $M$. Suppose that $\operatorname{dim} V=m>0$. Then

$$
\sigma_{1}+\sigma_{2}+\cdots+\sigma_{m} \leq \frac{\operatorname{Vol}(\partial M)}{\operatorname{Vol}(M)} .
$$

As a direct corollary of Theorem 1.1, we have

$$
\sigma_{1}+\sigma_{2}+\cdots+\sigma_{n} \leq \frac{\operatorname{Vol}(\partial \Omega)}{\operatorname{Vol}(\Omega)}
$$

for any bounded smooth domain $\Omega$ in $\mathbb{R}^{n}$. This estimate is sharp because the equality holds when $\Omega$ is a round ball. By using the CauchySchwartz inequality, one has

$$
\frac{1}{\sigma_{1}}+\frac{1}{\sigma_{2}}+\cdots+\frac{1}{\sigma_{n}} \geq \frac{n^{2} \operatorname{Vol}(\Omega)}{\operatorname{Vol}(\partial \Omega)}
$$

However, this estimate is weaker than Brock's inverse trace estimate [2]:

$$
\frac{1}{\sigma_{1}}+\frac{1}{\sigma_{2}}+\cdots+\frac{1}{\sigma_{n}} \geq \frac{n \operatorname{Vol}^{\frac{1}{n}}(\Omega)}{\operatorname{Vol}^{\frac{1}{n}}\left(\mathbb{B}^{n}\right)}
$$

because of the isoperimetric inequality for bounded Euclidean domains. 
In this paper, we characterize the equality case of (1.3). In summary, combining with Theorem 1.1, we have the following result.

Theorem 1.2. Let $\left(M^{n}, g\right)$ be a compact Riemannian manifold with nonempty boundary and $V$ be the space of parallel exact 1-forms on $M$. Suppose that $\operatorname{dim} V=m>0$. Then

$$
\sigma_{1}+\sigma_{2}+\cdots+\sigma_{m} \leq \frac{\operatorname{Vol}(\partial M)}{\operatorname{Vol}(M)} .
$$

The equality holds if and only if $M$ is a metric product of $\mathbb{B}^{m}(R)$ and a closed manifold $F$ with $R=\frac{m \operatorname{Vol}(M)}{\operatorname{Vol}(\partial M)}$ and

$$
\sigma\left(\mu_{1}(F)\right) \geq \frac{1}{R}
$$

where $\mu_{1}(F)$ is the first positive eigenvalue for the Laplacian operator on $F$ and

$$
\sigma(\mu):=\inf _{f \in C^{\infty}\left(\mathbb{B}^{m}(R)\right)} \frac{\int_{\mathbb{B}^{m}(R)}\left(\|\nabla f\|^{2}+\mu f^{2}\right) d V_{\mathbb{B}^{m}(R)}}{\int_{\partial \mathbb{B}^{m}(R)} f^{2} d V_{\partial \mathbb{B}^{m}(R)}}
$$

which is the first eigenvalue of the following boundary value problem:

$$
\begin{cases}\Delta f=\mu f & \text { on } \mathbb{B}^{m}(R) \\ \frac{\partial f}{\partial \nu}=\sigma f & \text { on } \partial \mathbb{B}^{m}(R) .\end{cases}
$$

The restriction (1.8) is necessary. For example, consider $M=[-1,1] \times$ $\mathbb{S}^{1}(L)$ where $\mathbb{S}^{1}(L)$ is the round circle with radius $L$. As computed in [4],

$$
\sigma_{1}(M)=\min \left\{\sigma\left(\frac{1}{L^{2}}\right), 1\right\}=\min \left\{\frac{1}{L} \tanh \left(\frac{1}{L}\right), 1\right\}
$$

So,

$$
\sigma_{1}(M)=\frac{\operatorname{Vol}(\partial M)}{\operatorname{Vol}(M)}=1
$$

only when $\frac{1}{L} \tanh \left(\frac{1}{L}\right) \geq 1$.

Moreover, as a direct corollary of Theorem 1.2 , one has

Corollary 1.1. Let $\Omega \subset \mathbb{R}^{n}$ be a bounded domain with smooth boundary. Then,

$$
\sigma_{1}+\sigma_{2}+\cdots+\sigma_{n} \leq \frac{\operatorname{Vol}(\partial \Omega)}{\operatorname{Vol}(\Omega)} .
$$

The equality holds if and only if $\Omega$ is a round ball. 
The key ingredient in the proof of the rigidity in Theorem 1.2 is the following result about triviality of a flat and totally geodesic Riemannian submersion between two complete Riemannian manifolds with boundary. Here a Riemanian submersion $\pi: M \rightarrow N$ is said to be flat if the horizontal distribution is integrable, and is said to be totally geodesic if each fibre is totally geodesic (see [17]).

Theorem 1.3. Let $\pi:\left(\bar{M}^{n+r}, \bar{g}\right) \rightarrow\left(M^{n}, g\right)$ be a flat and totally geodesic Riemannian submersion between two complete Riemannian manifolds with boundary such that $\pi(\partial \bar{M})=\partial M$. Suppose that $M$ is simply connected. Then, there is a complete Riemanian manifold $F$ without boundary and an isometry $\varphi: \bar{M} \rightarrow F \times M$ such that $\pi_{M} \circ \varphi=\pi$.

Note that the result fails when $M$ is not simply connected. For example, let $M$ be the annulus $\left\{x \in \mathbb{R}^{2} \mid 1 \leq\|x\| \leq 2\right\}$ with standard metric and $\bar{M}$ be its universal cover, then the conclusion fails. Moreover, it is not hard to see that the horizontal and vertical distributions of a flat and totally geodesic submersion are both parallel (see [17]). The assumption $\pi(\partial \bar{M})=\partial M$ implies that normal vectors on $\partial \bar{M}$ must be horizontal. So, by the deRham decompositions for Riemannian manifolds with boundary by the second named author [18], we have the splitting conclusion when $\bar{M}$ is simply connected. Theorem 1.3 just gives us a splitting conclusion by replacing the simply connectedness of $\bar{M}$ by the simply connectedness of $M$ which is more suitable for our application in the proof of Theorem 1.2 .

\section{Proofs of MAin Results}

Let's first recall the notion of development which will be used the in the proof of Theorem 1.3. Let $v:[0, T] \rightarrow T_{p} M$ be a curve in $T_{p} M$. A curve $\gamma:[0, T] \rightarrow M$ with

$$
\gamma^{\prime}(t)=P_{0}^{t}(\gamma)(v(t)) \text { and } \gamma(0)=p
$$

is called the development of $v$. This notion was presented in the language of principal fibre bundle in [8]. A proof of the local existence and uniqueness of developments can be found in [18].

Proof of Theorem 1.3. Let $p \in \operatorname{Int} M$ be a fixed point and $F=\pi^{-1}(p)$ where $\operatorname{Int} M=M \backslash \partial M$. Because, $\pi(\partial \bar{M})=\partial M, F$ is a complete Riemanian manifold without boundary. For each $q \in M$, let $\gamma:[0,1] \rightarrow$ $M$ be a curve such that $\gamma(0)=p$ and $\gamma(1)=q$. For each $\bar{p} \in F$, let $\bar{\gamma}_{\bar{p}}$ be the horizontal lift of $\gamma$ with $\bar{\gamma}_{\bar{p}}(0)=\bar{p}$. Consider the map $\Phi_{\gamma}: F \rightarrow \pi^{-1}(q)$ sending $\bar{p} \rightarrow \bar{\gamma}_{\bar{p}}(1)$. It is not hard to see that $\Phi_{\gamma}$ is an isometry (see [10]). To show the conclusion of the theorem, we only 
need to prove that $\Phi_{\gamma}$ is independent of the choice of $\gamma$ and depending only on the end point $q$ (see [10, Thoerem 5]).

Let $\gamma_{0}, \gamma_{1}:[0,1] \rightarrow M$ be two smooth curves with $\gamma_{0}(0)=\gamma_{1}(0)=p$ and $\gamma_{0}(1)=\gamma_{1}(1)=q$. Because $M$ is simply connected, there is a homotopy $\Psi:[0,1] \times[0,1] \rightarrow M$ such that

$$
\left\{\begin{array}{l}
\Psi(0, t)=\gamma_{0}(t) \\
\Psi(1, t)=\gamma_{1}(t) \\
\Psi(u, 0)=p \\
\Psi(u, 1)=q .
\end{array}\right.
$$

For each $\bar{p} \in F$, let $\bar{\Psi}_{\bar{p}}$ be the horizontal lift of $\Psi$ with $\bar{\Psi}_{\bar{p}}(u, 0)=\bar{p}$. Let $v(u, t) \in T_{p} M$ be given by

$$
v(u, t)=P_{t}^{0}\left(\gamma_{u}\right)\left(\gamma_{u}^{\prime}(t)\right)
$$

where $\gamma_{u}(t)=\Psi(u, t)$. Then, $\gamma_{u}$ is the development of $v(u, \cdot)$. Let $\bar{v}(u, t) \in T_{\bar{p}} \bar{M}$ be the horizontal lift of $v(u, t)$. Because $\pi: \bar{M} \rightarrow M$ is locally splitting (see [17]), $\bar{\Psi}_{\bar{p}}(u, \cdot)$ is the development of $\bar{v}(u, \cdot)$.

Let $e_{1}, e_{2}, \cdots, e_{n}$ be an orthonormal frame of $T_{p} M$ and $\bar{e}_{i} \in T_{\bar{p}} \bar{M}$ be the horizontal lift of $e_{i}$ for $i=1,2, \cdots, n$. Let $E_{i}(u, t)=P_{0}^{t}\left(\gamma_{u}\right)\left(e_{i}\right)$ and $\bar{E}_{i}(u, t)=P_{0}^{t}\left(\bar{\gamma}_{u}\right)\left(\bar{e}_{i}\right)$ where $\bar{\gamma}_{u}(t)=\bar{\Psi}_{\bar{p}}(u, t)$. Because $\pi$ is locally splitting again, $\bar{E}_{i}(u, t)$ is the horizontal lift of $E_{i}(u, t)$. Let $\bar{e}_{n+1}, \cdots, \bar{e}_{n+r} \in$ $T_{\bar{p}} F$ be an orthonormal frame and $\bar{E}_{\alpha}(u, t)=P_{0}^{t}\left(\bar{\gamma}_{u}\right)\left(\bar{e}_{\alpha}\right)$ for $\alpha=$ $n+1, \cdots, n+r$. By parallel-ness of the vertical distribution, $\bar{E}_{\alpha}(u, t)$ is vertical.

Suppose that

$$
\frac{\partial \Psi}{\partial u}=\sum_{i=1}^{n} U_{i} E_{i}
$$

and

$$
v=\sum_{i=1}^{n} v_{i} e_{i} .
$$


Then, by [18], $U_{i}$ 's satisfy the following Cauchy problem:

$$
\begin{cases}U_{i}^{\prime \prime}=\sum_{j, k, l=1}^{n} v_{k} v_{l} R\left(E_{k}, E_{i}, E_{l}, E_{j}\right) U_{j}+\partial_{u} \partial_{t} v_{i}+\sum_{j=1}^{n} \partial_{t} v_{j} X_{j i} & i=1,2, \cdots, n \\ X_{i j}^{\prime}=\sum_{k, l=1}^{n} v_{l} R\left(E_{i}, E_{j}, E_{l}, E_{k}\right) U_{k} & i, j=1,2, \cdots, n \\ X_{i j}(u, 0)=0 & i, j=1,2, \cdots, n \\ U_{i}(u, 0)=0 & i=1,2, \cdots, n \\ U_{i}^{\prime}(u, 0)=\partial_{u} v_{i}(u, 0) & i=1,2, \cdots, n .\end{cases}
$$

Here the symbol' means taking derivative with respect to $t$ and $R$ is the curvature tensor of $M$.

Suppose that

$$
\frac{\partial \bar{\Psi}_{\bar{p}}}{\partial u}=\sum_{a=1}^{n+r} \bar{U}_{a} \bar{E}_{a}
$$

and note that

$$
\bar{v}=\sum_{i=1}^{n} v_{i} \bar{e}_{i} .
$$

So, by [18 again, $\bar{U}_{a}$ 's satisfy the following Cauchy problem:

$$
\begin{cases}\bar{U}_{a}^{\prime \prime}=\sum_{b, c, d=1}^{n+r} v_{c} v_{d} \bar{R}\left(\bar{E}_{c}, \bar{E}_{a}, \bar{E}_{d}, \bar{E}_{b}\right) \bar{U}_{b}+\partial_{u} \partial_{t} v_{a}+\sum_{b=1}^{n+r} \partial_{t} v_{b} \bar{X}_{b a} & a=1,2, \cdots, n+r \\ \bar{X}_{a b}^{\prime}=\sum_{c, d=1}^{n+r} v_{d} \bar{R}\left(\bar{E}_{a}, \bar{E}_{b}, \bar{E}_{d}, \bar{E}_{c}\right) U_{c} & a, b=1,2, \cdots, n+r \\ \bar{X}_{a b}(u, 0)=0 & a, b=1,2, \cdots, n+r \\ \bar{U}_{a}(u, 0)=0 & a=1,2, \cdots, n+r \\ \bar{U}_{a}^{\prime}(u, 0)=\partial_{u} v_{a}(u, 0) & a=1,2, \cdots, n+r\end{cases}
$$

Here $\bar{R}$ is curvature tensor of $\bar{M}$ and we take $v_{\alpha}=0$ for $\alpha=n+1, n+$ $2, \cdots, n+r$.

Because $\pi$ is local splitting,

$$
\begin{gathered}
\bar{R}\left(\bar{E}_{i}, \bar{E}_{j}, \bar{E}_{k}, \bar{E}_{l}\right)=R\left(E_{i}, E_{j}, E_{k}, E_{l}\right), \\
\bar{R}\left(\bar{E}_{\alpha}, \bar{E}_{j}, \bar{E}_{k}, \bar{E}_{l}\right)=0
\end{gathered}
$$

and

$$
\bar{R}\left(\bar{E}_{\alpha}, \bar{E}_{\beta}, \bar{E}_{k}, \bar{E}_{l}\right)=0
$$


for $i, j, k, l=1,2, \cdots, n$ and $\alpha, \beta=n+1, \cdots, n+r$. So, it is not hard to check that

$$
\left\{\begin{array}{l}
\bar{U}_{i}=U_{i} \\
\bar{U}_{\alpha}=0 \\
\bar{X}_{i j}=X_{i j} \\
\bar{X}_{i \alpha}=\bar{X}_{\alpha i}=\bar{X}_{\alpha \beta}=0
\end{array}\right.
$$

is the solution of the Cauchy problem (2.9). Therefore,

$$
\frac{\partial \bar{\Psi}_{\bar{p}}}{\partial u}(u, 1)=0
$$

and $\Phi_{\gamma_{0}}=\Phi_{\gamma_{1}}$. This completes the proof of the theorem.

We are now ready to prove Theorem 1.2. For completeness, we will also present a proof of the estimate (1.7) which uses the idea of Raulot and Savo in [11, 12] and is not the same as our previous proof in [13].

Proof of Theorem 1.2. Note that for any harmonic function $f$ on $M$, one has

$$
\begin{aligned}
\left(\int_{M}\|\nabla f\|^{2} d V_{M}\right)^{2} & =\left(\int_{M} \operatorname{div}(f \nabla f) d V_{M}\right)^{2} \\
& =\left(\int_{\partial M} f \frac{\partial f}{\partial \nu} d V_{\partial M}\right)^{2} \\
& \leq \int_{\partial M} f^{2} d V_{\partial M} \int_{\partial M}\left(\frac{\partial f}{\partial \nu}\right)^{2} d V_{\partial M}
\end{aligned}
$$

Equality holds if and only if $f$ is a Steklov eigenfunction. So, for any non-trivial harmonic function $f$ on $M$,

$$
\frac{\int_{M}\|\nabla f\|^{2} d V_{M}}{\int_{\partial M} f^{2} d V_{\partial M}} \leq \frac{\int_{\partial M}\left(\frac{\partial f}{\partial \nu}\right)^{2} d V_{\partial M}}{\int_{M}\|\nabla f\|^{2} d V_{M}} .
$$

Let

$$
U=\left\{f \in C^{\infty}(M) \mid \int_{\partial M} f d V_{\partial M}=0, d f \text { is paralell. }\right\} .
$$

By assumption, we know that $\operatorname{dim} U=m$. By the inequality (2.16) and Courant's min-max principle, we have

$$
\sigma_{k} \leq \lambda_{k}
$$

for any $k=1,2, \cdots, m$, where $\lambda_{1} \leq \lambda_{2} \leq \cdots \leq \lambda_{m}$ are the eigenvalues of the Rayleigh quotient $\frac{\int_{\partial M}\left(\frac{\partial f}{\partial \nu}\right)^{2} d V_{\partial M}}{\int_{M}\|\nabla f\|^{2} d V_{M}}$ restricted on $U$. 
Let $f_{1}, f_{2}, \cdots, f_{m}$ be a basis of $U$ such that

$$
\left\langle\nabla f_{i}, \nabla f_{j}\right\rangle \equiv \delta_{i j}
$$

and $f_{i}$ is the eigenfunction of the Rayleigh quotient $\frac{\int_{\partial M}\left(\frac{\partial f}{\partial \nu}\right)^{2} d V_{\partial M}}{\int_{M}\|\nabla f\|^{2} d V_{M}}$ restricted on $U$ with respect to the eigenvalue $\lambda_{i}$. Then

$$
\begin{aligned}
\sigma_{1}+\sigma_{2}+\cdots+\sigma_{m} & \leq \lambda_{1}+\lambda_{2}+\cdots+\lambda_{m} \\
& =\frac{\int_{\partial M} \sum_{i=1}^{m}\left(\frac{\partial f_{i}}{\partial \nu}\right)^{2} d V_{\partial M}}{\operatorname{Vol}(M)} \\
& =\frac{\int_{\partial M} \sum_{i=1}^{m}\left\langle\nu, \nabla f_{i}\right\rangle^{2} d V_{\partial M}}{\operatorname{Vol}(M)} \\
& \leq \frac{\int_{\partial M}\|v\|^{2} d V_{\partial M}}{\operatorname{Vol}(M)} \\
& =\frac{\operatorname{Vol}(\partial M)}{\operatorname{Vol}(M)} .
\end{aligned}
$$

When the equality holds, one has $\nu \in \operatorname{span}\left\{\nabla f_{1}, \nabla f_{2}, \cdots, \nabla f_{m}\right\}$ and $f_{1}, f_{2}, \cdots, f_{m}$ are the Steklov eigenfunctions with respect to $\sigma_{1}, \sigma_{2}, \cdots, \sigma_{m}$ respectively. So,

$$
\nu=\sum_{i=1}^{m}\left\langle\nu, \nabla f_{i}\right\rangle \nabla f_{i}=\sum_{i=1}^{m} \sigma_{i} f_{i} \nabla f_{i} .
$$

Therefore,

$$
\sum_{i=1}^{m} \sigma_{i}^{2} f_{i}^{2}=\|\nu\|^{2}=1
$$

on $\partial M$. This implies that $\nu$ must be parallel to

$$
\nabla\left(\sum_{i=1}^{m} \sigma_{i}^{2} f_{i}^{2}\right)=2 \sum_{i=1}^{m} \sigma_{i}^{2} f_{i} \nabla f_{i} .
$$

By comparing (2.20) and (2.22), we have

$$
\sigma_{1}=\sigma_{2}=\cdots=\sigma_{m}=\frac{\operatorname{Vol}(\partial M)}{m \operatorname{Vol}(M)}
$$

Then, by (2.21),

$$
\sum_{i=1}^{m} f_{i}^{2}=\left(\frac{m \operatorname{Vol}(M)}{\operatorname{Vol}(\partial M)}\right)^{2}=R^{2}
$$

on $\partial M$. Let $\pi=\left(f_{1}, f_{2}, \cdots, f_{m}\right)$. It is not hard to check that $\pi$ is a Riemannian submersion from $M$ to $\mathbb{B}^{m}(R)$ with $\pi(\partial M)=\partial \mathbb{B}^{m}(R)$. 
By Theorem [1.3, we know that $M=\mathbb{B}^{m}(R) \times F$ for some closed Riemannian manifold $F$.

Moreover, when $M=\mathbb{B}^{m}(R) \times F$ with $F$ a closed Riemannian manifold. Let

$$
0=\mu_{0}<\mu_{1} \leq \mu_{2} \leq \cdots \leq \mu_{k} \leq \cdots
$$

be the spectrum of the Laplacian operator for $F$. Then, by a standard argument as in [4] using the method of separating variables, we know that the Steklov spectrum of $M$ is formed by the eigenvalues $\sigma$ of the following boundary value problems on $\mathbb{B}^{m}(R)$ :

$$
\begin{cases}\Delta f=\mu_{i} f & \text { on } \mathbb{B}^{m}(R) \\ \frac{\partial f}{\partial \nu}=\sigma f & \text { on } \partial \mathbb{B}^{m}(R)\end{cases}
$$

for $i=0,1,2, \cdots$. The corresponding Rayleigh quotient of the eigenvalue problem (2.25) is

$$
Q_{i}(f)=\frac{\int_{M}\left(\|\nabla f\|^{2}+\mu_{i} f^{2}\right) d V_{M}}{\int_{\partial M} f^{2} d V_{\partial M}}
$$

for $i=0,1,2, \cdots$. So, it is not hard to see that

$$
\sigma_{1}(M)=\cdots=\sigma_{m}(M)=\sigma_{1}\left(\mathbb{B}^{m}(R)\right)=\cdots=\sigma_{m}\left(\mathbb{B}^{m}(R)\right)=\frac{1}{R}
$$

which is the first $m$ positive Steklov eigenvalues of $M$ only when

$$
\sigma\left(\mu_{1}\right) \geq \frac{1}{R}
$$

because

$$
\sigma\left(\mu_{1}\right) \leq \sigma\left(\mu_{2}\right) \leq \cdots \leq \sigma\left(\mu_{k}\right) \leq \cdots .
$$

This completes the proof of the theorem.

\section{REFERENCES}

[1] Belishev M., Sharafutdinov V. Dirichlet to Neumann operator on differential forms. Bull. Sci. Math. 132 (2008), no. 2, 128-145.

[2] Brock F. An isoperimetric inequality for eigenvalues of the Stekloff problem. Z. Angew. Math. Mech. 81 (2001), no. 1, 69-71.

[3] Calderón A.-P. On an inverse boundary value problem. Seminar on Numerical Analysis and its Applications to Continuum Physics (Rio de Janeiro, 1980), pp. 65-73, Soc. Brasil. Mat., Rio de Janeiro, 1980.

[4] Fraser Ailana, Schoen Richard. The first Steklov eigenvalue, conformal geometry, and minimal surfaces, Adv. Math. 226 (2011), no. 5, 4011-4030.

[5] Fraser Ailana, Schoen Richard. Sharp eigenvalue bounds and minimal surfaces in the ball. Invent. Math. 203 (2016), no. 3, 823-890.

[6] Hua Bobo, Huan Yan, Wang Zuoqin. First eigenvalue estimates of Dirichletto-Neumann operators on graphs. Calc. Var. Partial Differential Equations 56 (2017), no. 6, Art. 178, 21 pp. 
[7] Joshi M. S., Lionheart W. R. B. An inverse boundary value problem for harmonic differential forms. Asymptot. Anal. 41 (2005), no. 2, 93-106.

[8] Kobayashi Shoshichi, Nomizu Katsumi. Foundations of differential geometry. Vol. I. Reprint of the 1963 original. Wiley Classics Library. A WileyInterscience Publication. John Wiley \& Sons, Inc., New York, 1996. xii+329 pp.

[9] Kuznetsov Nikolay, Kulczycki Tadeusz, Kwaśnicki Mateusz, Nazarov Alexander, Poborchi Sergey, Polterovich Iosif, Siudeja Bartłomiej. The legacy of Vladimir Andreevich Steklov. Notices Amer. Math. Soc. 61 (2014), no. 1, 9-22.

[10] O'Neill Barrett. The fundamental equations of a submersion. Michigan Math. J. 13 (1966), 459-469.

[11] Raulot S., Savo A. On the spectrum of the Dirichlet-to-Neumann operator acting on forms of a Euclidean domain. J. Geom. Phys. 77 (2014), 1-12.

[12] Raulot S., Savo A. On the first eigenvalue of the Dirichlet-to-Neumann operator on forms. J. Funct. Anal. 262 (2012), no. 3, 889-914.

[13] Shi Yongjie, Yu Chengjie. Trace and inverse trace of Steklov eigenvalues. J. Differential Equations 261 (2016), no. 3, 2026-2040.

[14] Shi Yongjie, Yu Chengjie. Higher order Dirichlet-to-Neumann maps on graphs and their eigenvalues. arXiv:math.DG/1904.03880.

[15] Stekloff W. Sur les problèmes fondamentaux de la physique mathématique. Ann. Sci. École Norm. Sup. (3) 19 (1902), 191-259.

[16] Ulmann G. Electrical impedance tomography and Calderons problem http://www.math.washington.edu/ gunther/publications $\quad / \mathrm{Pa}-$ pers/calderoniprevised.pdf

[17] Walschap Gerard. Metric foliations and curvature. J. Geom. Anal. 2 (1992), no. 4, 373-381.

[18] Yu Chengjie. De Rham decomposition for Riemannian manifolds with boundary. arXiv:math.DG/1911.04066.

Department of Mathematics, Shantou University, Shantou, GuangDONG, 515063, CHINA

E-mail address: yjshi@stu.edu.cn

Department of Mathematics, Shantou University, Shantou, GuangDONG, 515063, CHINA

E-mail address: cjyu@stu.edu.cn 